\title{
Pilas de combustible de una sola cámara soportadas sobre el ánodo y basadas en electrolitos de ceria dopada con gadolinia
}

\author{
M. MORALES ${ }^{1,2}$, S. PIÑOL ${ }^{1}$, F. ESPIELL ${ }^{2}$ \\ ${ }^{1}$ Institut de Ciència de Materials de Barcelona, CSIC. \\ Campus de la Universitat Autònoma de Barcelona, Bellaterra, Barcelona, España. \\ ${ }^{2}$ DIOPMA / CeRMAE, Departament de Ciència dels Materials i Enginyeria Metal·lúrgica, Institut de Nanociència i Nanotecnologia de la \\ Universitat de Barcelona (IN2NUB). \\ Facultat de Química de la Universitat de Barcelona, Barcelona, España.
}

\begin{abstract}
La utilización de electrolitos soportados en el ánodo es una estrategia muy útil para mejorar las propiedades eléctricas de las pilas de combustible de óxido sólido, debido a que permiten disminuir considerablemente el espesor de los electrolitos. Para este trabajo, se han preparado exitosamente pilas de combustible de óxido sólido con electrolitos de ceria dopada con $\mathrm{Gd}, \mathrm{Ce}_{1-\mathrm{x}} \mathrm{Gd}_{\mathrm{x}} \mathrm{O}(\mathrm{CGO})$ soportados sobre un ánodo formado por un cermet de Ni/CGO. Dichas pilas se han instalado y caracterizado en un reactor de una sola cámara donde se ha hecho circular una mezcla de propano y aire. Para ello, se han preparado mezclas de polvos de $\mathrm{NiO}$ y de ceria dopada con gadolinia, con diferentes composiciones y tamaño de partículas, para obtener los ánodos con porosidades apropiadas y así utilizarlos como soporte del electrolito en las pilas. Los polvos de los electrolitos de CGO se han preparado por la técnica sol-gel y se han depositado por "dip coating" con diferentes espesores (15-30 $\mu \mathrm{m})$ utilizando una tinta preparada a partir de partículas nanométricas dispersadas en una resina comercial. Los cátodos de $\mathrm{La}_{1-\mathrm{x}} \mathrm{Sr}_{\mathrm{x}} \mathrm{CoO}_{3-\mathrm{S}}$ (LSCO) se han preparado también por la técnica sol-gel y se han depositado sobre la capa fina del electrolito. Finalmente, las propiedades eléctricas se han determinado en un reactor de una sola cámara dónde el propano se ha mezclado con aire sintético por encima del límite superior de inflamabilidad. En estas condiciones experimentales se han obtenido altas densidades de potencia estables, controlando las velocidades de flujo total de gas transportador y la presión parcial de propano.
\end{abstract}

Palabras clave: SOFC, monocámara, ceria, cobaltita, propano.

Anode-supported single-chamber SOFCs based on gadolinia doped ceria electrolytes

The utilization of anode supported electrolytes is a useful strategy to increase the electrical properties of the solid oxide fuel cells, because it is possible to decrease considerably the thickness of the electrolytes. We have prepared successfully singlechamber fuel cells of gadolinia doped ceria electrolytes $\mathrm{Ce}_{1-\mathrm{x}} \mathrm{Gd} \mathrm{O}_{2-\mathrm{y}}$ (CGO) supported on an anode formed by a cermet of $\mathrm{Ni}$-CGO. Mixtures of precursor powders of $\mathrm{NiO}$ and gadolinium doped ceria with different particle sizes and compositions were analyzed to obtain optimal bulk porous anodes to be used as anode supported fuel cells. Doped ceria electrolytes were prepared by sol-gel related techniques. Then, ceria based electrolytes were deposited by dip coating at different thickness $(15-30 \mu \mathrm{m})$ using an ink prepared with nanometric powders of electrolytes dispersed in a commercial liquid polymer. Cathodes of $\mathrm{La}_{1-x} \mathrm{Sr}_{x} \mathrm{CoO}_{3-5}$ (LSCO) were also prepared by sol-gel related techniques and were deposited by dip coating on the electrolyte thick films. Finally, electrical properties were determined in a single-chamber reactor where propane as fuel was mixed with synthetic air above the higher explosive limit. Stable density currents were obtained in these experimental conditions, but flow rates of the carrier gas and propane partial pressure were determinants for the optimization of the electrical properties of the fuel cells.

Keywords: SOFC, single-chamber, ceria, cobaltite, propane.

\section{INTRODUCCIÓN}

En los últimos años, se ha desarrollado un nuevo tipo de pila de combustible de óxido sólido que funciona en un reactor de una sola cámara y que presenta elevadas densidades de potencia a relativamente bajas temperaturas $\left(500-630^{\circ} \mathrm{C}\right)$. Este tipo de pilas de combustible basadas en una sola cámara han sido propuestas en estos últimos años por varios investigadores (1-7). La principal diferencia entre las pilas de combustible de una sola cámara y las convencionales de dos cámaras es que en las primeras los electrodos están simultáneamente en contacto con el combustible (hidrocarburo, alcohol, hidrógeno...) y el aire por encima del límite superior de inflamabilidad. La principal ventaja de este diseño es que, al no separarse los gases, se simplifica considerablemente la celda y se reduce su volumen, peso y coste, lo cual tiene un gran impacto tecnológico en la preparación de apilamientos ya que no se necesita materiales de sellado. Además, la caracterización obtenida puede extrapolarse a las pilas de combustible convencionales. Estas pilas de combustible pueden trabajar directamente 
con hidrocarburos o alcoholes (reformado interno) porque la temperatura de trabajo es óptima para el reformado de dichos combustibles y la conductividad iónica del electrolito. Además, el envenenamiento del ánodo por carbón o azufre no es posible debido a la presencia de aire sobre el ánodo que tiene un poder limpiador por oxidación.

Las pilas SOFC de una sola cámara cuya representación está en la figura 1, se basan en el empleo de catalizadores selectivos a las reacciones de cada electrodo: la reducción del oxígeno en el cátodo y la oxidación del combustible (hidrocarburo, alcohol...) en el ánodo, utilizando el $\mathrm{O}_{2}$ presente. Es interesante recalcar que debido al calor desprendido por la oxidación exotérmica del propano en el ánodo [ver reacción 2], la temperatura real de la pila es más alta que la del horno, dependiendo de las condiciones experimentales (9). Este autocalentamiento es muy superior al calor que proporciona la reacción de oxidación del hidrógeno [reacción 1] en las pilas
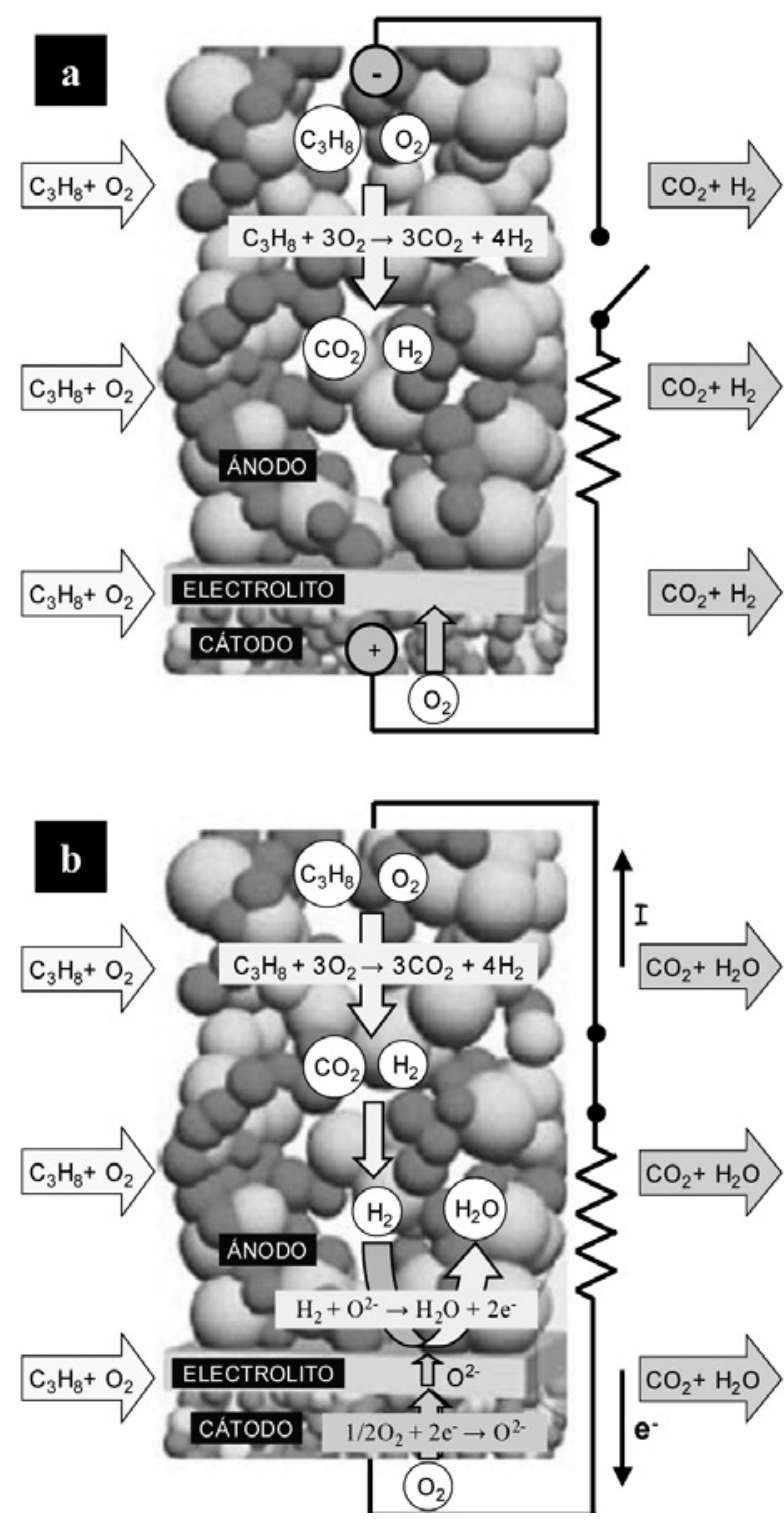

Fig. 1- Esquema del funcionamiento de la pila de una sola cámara. a) En circuito abierto. b) En circuito cerrado. de dos cámaras, lo cual explica el más alto valor de densidad de corriente aparente, que se ha encontrado a veces en la bibliografía (8), para los reactores de una cámara que para dos cámaras, a la misma temperatura de horno:

$$
\mathrm{H}_{2}(\mathrm{~g})+1 / 2 \mathrm{O}_{2}(\mathrm{~g}) \rightarrow \mathrm{H}_{2} \mathrm{O}(\mathrm{g}) \quad \Delta \mathrm{H}_{550^{\circ} \mathrm{C}}=-240 \mathrm{~kJ} \mathrm{~mol}^{-1}
$$

[Reacción 1]

$$
\mathrm{C}_{3} \mathrm{H}_{8}(\mathrm{~g})+5 \mathrm{O}_{2}(\mathrm{~g}) \rightarrow 3 \mathrm{CO}_{2}(\mathrm{~g})+4 \mathrm{H}_{2} \mathrm{O}(\mathrm{g}) \Delta \mathrm{H}_{550^{\circ} \mathrm{C}}=-2220 \mathrm{~kJ} \mathrm{~mol}^{-1}
$$

[Reacción 2]

Los electrolitos de óxido sólido basados en la ceria dopada están considerados como uno de los candidatos más prometedores para usarlos en las pilas de combustible de baja temperatura porque presentan una conductividad iónica más elevada que el YSZ entre 400 y $700^{\circ} \mathrm{C}$. La substitución de $\mathrm{Ce}^{4+}$ por cationes trivalentes como el $\mathrm{Gd}^{3+}, \mathrm{Sm}^{3+}, \mathrm{Y}^{3+} \mathrm{o} \mathrm{La}^{3+}$ aumenta la estabilidad química y la conductividad iónica dificultando la reducibilidad de la ceria. Las substituciones más efectivas son el $\mathrm{Gd}_{2} \mathrm{O}_{3}$ y $\mathrm{Sm}_{2} \mathrm{O}_{3}$ posiblemente debido al hecho de que minimizan el cambio del parámetro de celda.

En este tipo de pilas de combustible de una sola cámara, el ánodo y el cátodo están expuestos a la misma mezcla de combustible y aire, por esta razón existe cierta dificultad de encontrar cátodos estables en estas condiciones reductoras. Hibino et al (8) han publicado un alto valor relativo de densidad de potencia a $500^{\circ} \mathrm{C}$ para una pila de combustible soportada en el electrolito utilizando $\mathrm{Sm}_{0.5} \mathrm{Sr}_{0.5} \mathrm{CoO}_{3-\delta}$ mezclado con $\mathrm{Ce}_{0.8} \mathrm{Sm}_{0.2} \mathrm{O}_{1.9}(\mathrm{SSC}+\mathrm{CSO})$ como cátodo y con etano como combustible. Sin embargo, encontraron que este cátodo era incompatible con el propano para temperaturas superiores a $450^{\circ} \mathrm{C}$.

Por otra parte, Shao et al presentan un nuevo cátodo de composición: $\mathrm{Ba}_{0.5} \mathrm{Sr}_{0.5} \mathrm{Co}_{0.8} \mathrm{Fe}_{0.2} \mathrm{O}_{3-8}$ que presenta buen funcionamiento con propano en una pila de combustible de una sola cámara soportada sobre el ánodo y basada en electrolitos de CSO (10). Además, ellos confirman que el $\mathrm{Ce}_{0.8} \mathrm{Sm}_{0.2} \mathrm{O}_{1.9}$ no es estable en las mismas condiciones experimentales. Sin embargo, nosotros mostramos en el presente trabajo que el cátodo LSCO es estable hasta $640^{\circ} \mathrm{C}$ en una pila de combustible de una sola cámara con propano diluido en aire como combustible y un electrolito de CGO, de $\sim 20 \mu \mathrm{m}$ de espesor, soportado sobre un ánodo de Ni/CGO. También hemos encontrado que las pilas de combustible con LSCO como cátodo son también estables con CSO como electrolito en las mismas condiciones experimentales con propano como combustible.

Algunos autores han publicado que la ceria dopada con gadolinia (CGO) posee el valor más alto de conductividad iónica (11), lo cual ha sido confirmado por simulaciones teóricas basadas en una combinación de interacciones de Coulomb y relajaciones de la celda elemental (12). Esta interacción depende del tamaño del catión trivalente y las simulaciones sugieren que el radio catiónico trivalente en la ceria corresponde aproximadamente al del $\mathrm{Gd}^{3+}$. Sin embargo, otros autores han publicado valores más altos de conductividad iónica para el CSO $(13,14)$. Algunas de las diferencias entre los resultados presentados por los diferentes 
autores pueden ser debidas a la preparación de los polvos y a los efectos correspondientes a la resistencia del límite de grano $(15,16)$. Por estas razones, cabe esperar mejoras en las propiedades eléctricas optimizando el método de preparación de las muestras. En este trabajo presentamos los resultados obtenidos con el CGO pero hemos encontrado resultados similares para el CSO.

\section{EXPERIMENTAL}

\subsection{Síntesis de los electrodos y electrolito}

Se han preparado polvos con tamaño de partícula nanométrico de composición $60-80 \%$ de $\mathrm{NiO}$ y del $40-20 \%$ de $\mathrm{Ce}_{1-\mathrm{x}} \mathrm{Gd}_{\mathrm{x}} \mathrm{O}_{2-\mathrm{y}}(\mathrm{CGO})$, respectivamente para la preparación de los ánodos y de $\mathrm{La}_{1-\mathrm{x}} \mathrm{Sr}_{\mathrm{x}} \mathrm{CoO}_{3}$ (LSCO) para la preparación de los cátodos, por el método sol-gel de la acrilamida y por el método sol-gel del acetil-acetonato, respectivamente. Para ello, se ha partido en ambos casos de las soluciones de nitratos de los diferentes cationes. Los polvos de NiO-CGO se han preparado simultáneamente por coprecipitación y calcinación para obtener una mezcla homogénea de las dos fases con un tamaño de grano lo más pequeño posible. Todas estas muestras se han caracterizado por difracción de rayos $X$ y se ha visto que no presentan fases secundarias.

Los polvos del electrolito $\left(\mathrm{Ce}_{0.8} \mathrm{Gd}_{0.2} \mathrm{O}_{2-\mathrm{y}}\right)$ se han preparado por el método sol-gel del acetil-acetonato partiendo de los acetatos $\mathrm{Ce}\left(\mathrm{C}_{2} \mathrm{H}_{3} \mathrm{O}_{2}\right)_{3} \cdot 1.5 \mathrm{H}_{2} \mathrm{O}$ (Alfa Aesar, $99.9 \%$ pureza) y $\mathrm{Gd}\left(\mathrm{C}_{2} \mathrm{H}_{3} \mathrm{O}_{2}\right) \cdot \mathrm{xH}_{2} \mathrm{O}$ (Alfa Aesar, $99.9 \%$ pureza) como se describe en la referencia (16). Finalmente, se ha añadido un $2 \%$ en peso de $\mathrm{Co}\left(\mathrm{C}_{2} \mathrm{H}_{3} \mathrm{O}_{2}\right)_{3}$ para reducir la temperatura de densificación del electrolito depositado sobre el ánodo como se describe en un trabajo precedente (17).

\subsection{Preparación del ánodo y del electrolito}

Las pilas de combustible que se han estudiado en este trabajo se han soportado sobre el ánodo. Para ello, se han preparado discos de $\mathrm{NiO}$ y CGO $(80-20 \%, 70-30 \%$ y $60-40 \%$ respectivamente) de $10 \mathrm{~mm}$ de diámetroy $0,5 \mathrm{~mm}$ de espesor por prensado uniaxial a $3 \mathrm{Tn} / \mathrm{cm}^{2}$ durante 30 segundos. Después, se ha depositado el electrolito de CGO de $\sim 20 \mu \mathrm{m}$ de espesor por "dip coating" sobre los discos de NiO-CGO en verde, utilizando una tinta preparada con los polvos nanométricos de CGO $+2 \%$ Co dispersados en una resina comercial. Finalmente, los discos se han sinterizado entre 1350 y $1440^{\circ} \mathrm{C}$ durante 5 horas para producir la consolidación del ánodo y la densificación del electrolito de CGO, simultáneamente. El análisis microestrutural de las muestras sinterizadas de CGO se ha efectuado por SEM y se ha encontrado que la distribución de las fases de Ni y CGO que forman el ánodo es homogénea en las muestras de composición $60-40 \%$ y que la capa de electrolito presenta una alta densidad. La figura 2 muestra la variación de la porosidad en función de la composición de los cermets Ni-CGO obtenidos a partir de la reducción a $900^{\circ} \mathrm{C}$ en $\mathrm{Ar} / \mathrm{H}_{2} 5 \%$ de los composites $\mathrm{NiO}-\mathrm{CGO}$ correspondientes, para una temperatura de sinterización fijada en $1350^{\circ} \mathrm{C}$. El diámetro de los poros y el tamaño medio de los granos de Ni en el cermet Ni-CGO que va a ser utilizado como ánodo aumenta con el contenido de $\mathrm{NiO}$. Un crecimiento desmesurado de los granos de $\mathrm{Ni}$ ("coarsening") se observa en las muestras con un $80 \%$ de $\mathrm{NiO}$ y un $20 \%$ de CGO, mientras que la distribución más homogénea de las dos fases con un tamaño de grano más pequeño se observa en las muestras de composición 60-40\%. Así pues, las mejores propiedades eléctricas de los ánodos preparados se han encontrado para las muestras cercanas a dicha composición.
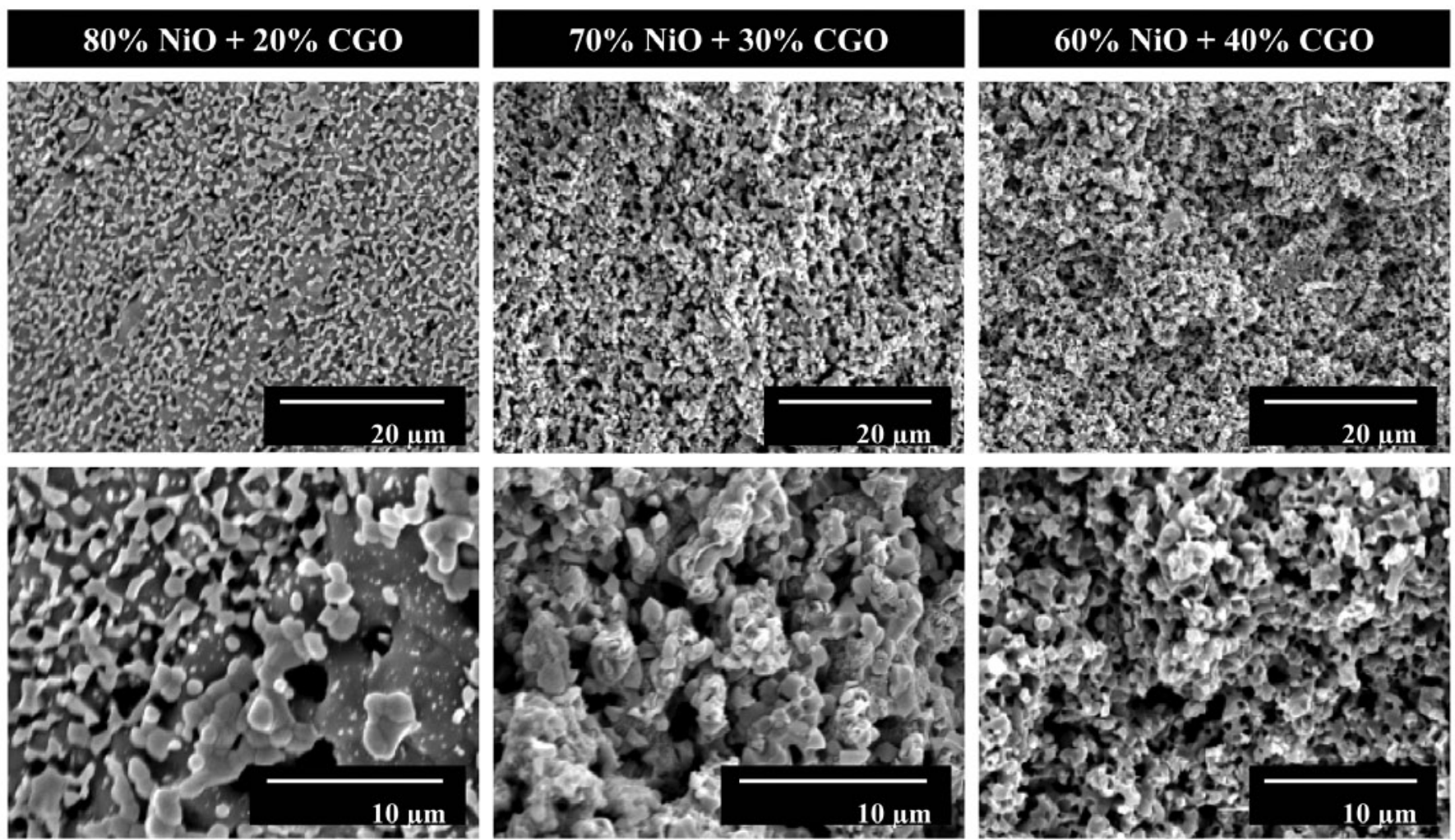

Fig. 2- Micrografías SEM correspondientes a ánodos "con diferentes composiciones de NiO-CGO" sinterizados a $1350^{\circ} \mathrm{C}$ durante 5 h. 
La densificación delelectrolito tienelugar, simultáneamente, durante la sinterización del ánodo en el mismo tratamiento térmico. La figura 3 muestra la variación del tamaño de grano del electrolito CGO con la temperatura. El diámetro de los granos de CGO se incrementa exponencialmente desde $1350^{\circ} \mathrm{C}$ hasta $1440^{\circ} \mathrm{C}$ y la densificación del electrolito no es óptima a temperaturas por debajo de $1340^{\circ} \mathrm{C}$. Como la máxima conductividad iónica del electrolito se alcanza cuando su tamaño de grano es lo más pequeño posible conservando una alta densidad, las propiedades eléctricas óptimas de las pilas de combustible se han encontrado en las muestras densificadas a temperaturas cercanas a $1350-1400^{\circ} \mathrm{C}$.
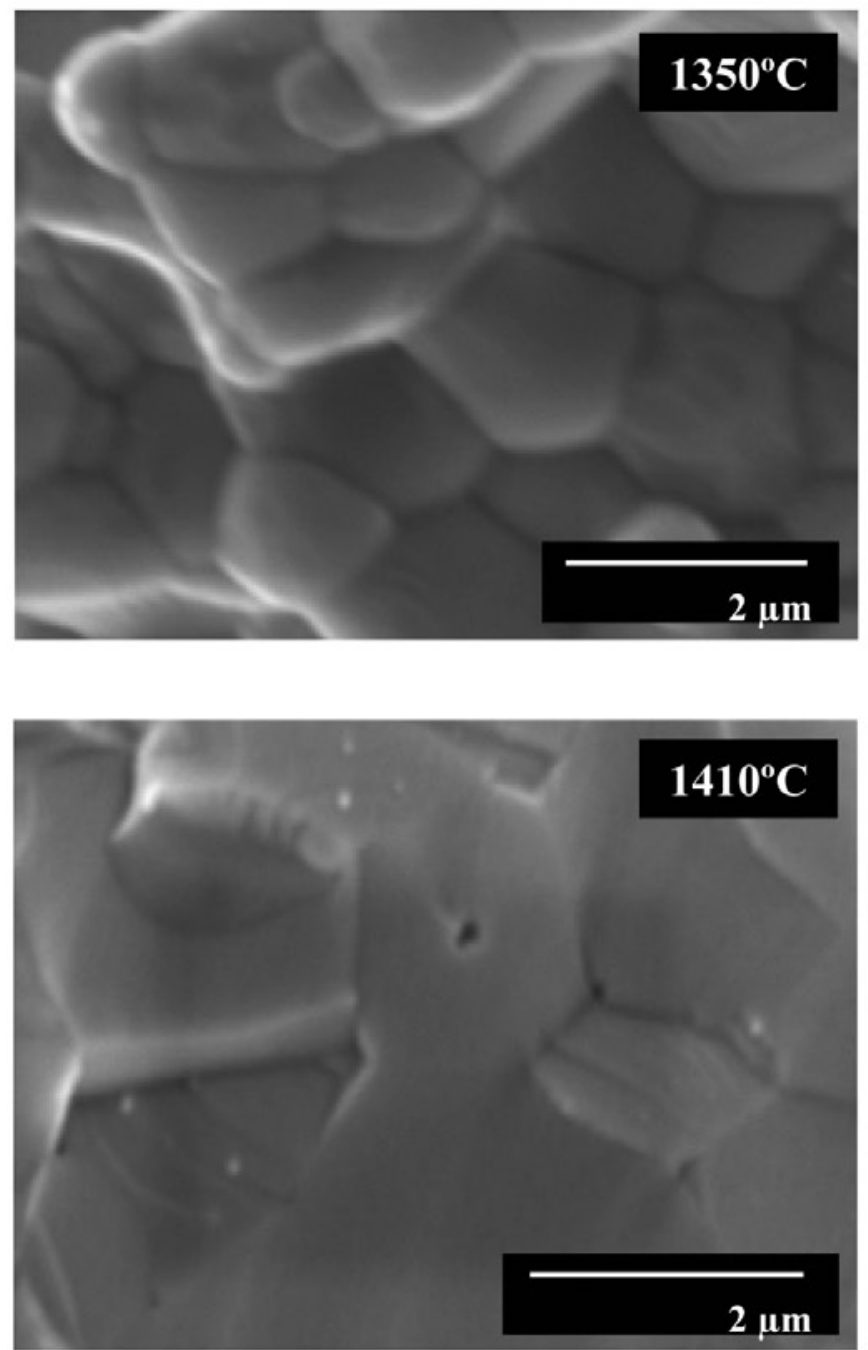

óptimos. En la figura 4, se ha representado detalladamente la estructura de una pila de combustible con el ánodo y electrolito preparados a $1350^{\circ} \mathrm{C}$ (fig. 4a). Las figuras $4 \mathrm{~b}$, c y d muestran la microestructura ampliada del electrolito, ánodo y cátodo, respectivamente. La porosidad en el cátodo es superior a la del ánodo debido a que la temperatura del tratamiento térmico del cátodo es inferior a la del ánodo $\left(640^{\circ} \mathrm{C}\right.$ frente a $\left.1350^{\circ} \mathrm{C}\right)$.

\subsection{Caracterización}

Los electrodos de la pila se han fijado a unos hilos de platino para recoger la corriente y medir sus propiedades
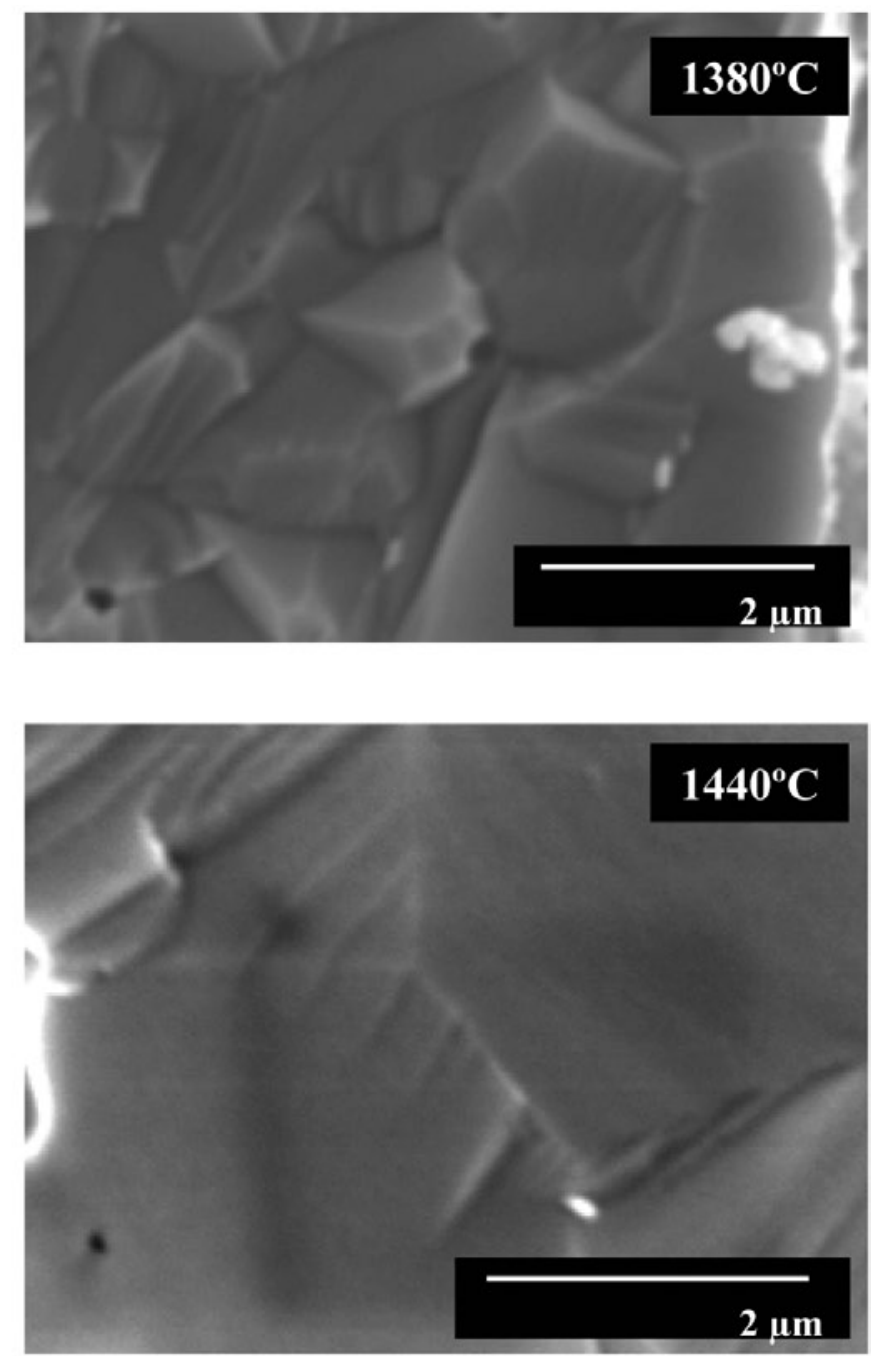

Fig. 3- Micrografías SEM correspondientes a electrolitos de CGO + 2\% de acetato de cobalto sinterizados sobre NiO-CGO (60-40\%, respectivamente) a diferentes temperaturas.

\subsection{Preparación del cátodo}

Los cátodos de LSCO $+10 \%$ de CGO en peso se han preparado a partir de los polvos obtenidos mediante los métodos sol-gel descritos anteriormente, mezclados en un mortero de ágata y depositados por "dip coating" directamente sobre la capa densa sinterizada del electrolito de CGO, usando una tinta con la misma resina comercial que el electrolito. La tinta del cátodo se ha tratado térmicamente junto con el ánodo y el electrolito a $640^{\circ} \mathrm{C}$ en el mismo horno y atmósfera dónde se realizan las medidas eléctricas para eliminar el componente orgánico de la tinta y conseguir unos contactos eléctricos eléctricas. Para fijar el cátodo se ha usado la misma tinta que se ha utilizado en la deposición de los electrodos. Para conseguir unos contactos eléctricos óptimos en los electrodos, ambas superficies se han pintado con las tintas de $\mathrm{Ni}+10 \%$ de CGO en peso para el ánodo y LSCO + 10\% de CGO en peso para el cátodo. La tinta de $\mathrm{Ni}$ se ha preparado a partir de un polvo de Ni comercial con una pureza del 99,9\% y 2,2-3,0 $\mu \mathrm{m}$ de tamaño medio de partícula. Por otra parte, se ha fijado un termopar adicional en el centro de la superficie del ánodo para determinar la temperatura real de la pila. La pila así fabricada 

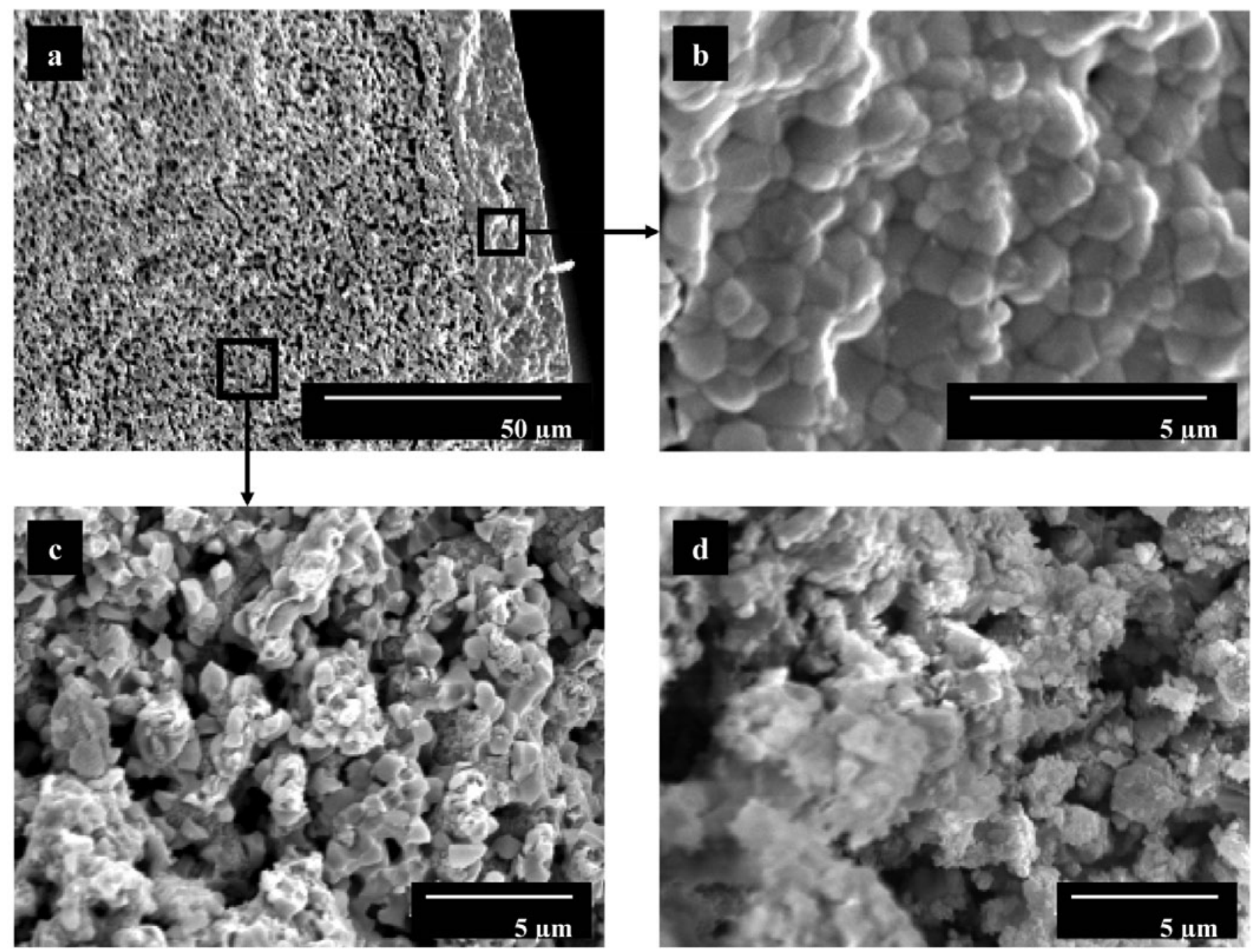

Fig. 4- Micrografías SEM de una pila de combustible de ánodo soportado con un electrolito de CGO de $15 \mu \mathrm{m}$ de espesor. a) Pila de combustible de ánodo soportado. b) Electrolito. c) Ánodo d) Cátodo.

se ha situado en el interior de un tubo de cuarzo de $23 \mathrm{~mm}$ de diámetro interno, dónde se ha hecho circular una mezcla de nitrógeno y oxígeno con la composición del aire $(80-20 \%$, respectivamente) a diferentes velocidades de flujo (250-480 $\mathrm{ml} / \mathrm{min}$ ). La influencia de la velocidad de flujo de propano en las medidas electroquímicas se ha analizado desde 0 a $40 \mathrm{ml} /$ min. El tubo de cuarzo se ha calentado mediante un horno tubular y las pilas de combustible han sido operativas entre $400^{\circ} \mathrm{C}$ y $650^{\circ} \mathrm{C}$.

La fuerza electromotriz de la pila se ha medido utilizando un electrómetro Keitley 617 con una resistencia de entrada de $10^{14} \Omega$. El valor de la densidad de corriente se ha determinado midiendo la caída de voltaje en una resistencia auxiliar conocida. Las curvas I-V se han determinado utilizando un equipo para medir la corriente y el voltaje con cargas variables conocidas. La temperatura del horno se ha fijado con un controlador Eurotherm y la temperatura de la pila se ha medido con un termopar fijado al ánodo de la muestra. La composición de los gases de salida del horno se ha determinado mediante un cromatógrafo de gases, Agilent 3000, con dos columnas independientes, una específica para detectar especies orgánicas tales como hidrocarburos $\left(\mathrm{CH}_{4}, \mathrm{C}_{3} \mathrm{H}_{8} \ldots\right), \mathrm{CO}, \mathrm{CO}_{2} \ldots$ y la segunda que detecta especies inorgánicas $\left(\mathrm{Ar}, \mathrm{O}_{2}, \mathrm{~N}_{2}, \mathrm{H}_{2} \ldots\right)$.

\section{RESULTADOS Y DISCUSIÓN}

La fracción orgánica de las tintas que contienen los polvos de los electrodos se elimina durante el calentamiento (aproximadamente a $\sim 300^{\circ} \mathrm{C}$ ) antes de llegar a la temperatura donde empieza la oxidación parcial del propano $\left(\sim 350^{\circ} \mathrm{C}\right)$. En la figura 6 se ha representado el análisis cromatográfico de la mezcla de gases que se obtiene a la salida del reactor. Se ha encontrado que la temperatura de la pila es aproximadamente la misma que la del horno durante el calentamiento hasta que empieza a reformarse el propano (aproximadamente a $\sim 350^{\circ} \mathrm{C}$ ). Entonces, aparece una fuerza electromotriz y una corriente eléctrica a $\mathrm{V} \sim 0$ y la temperatura de la pila aumenta drásticamente. La temperatura real de la pila alcanza unos $80^{\circ} \mathrm{C}$ más que la temperatura del horno, dependiendo de las condiciones experimentales tales como el aislamiento del horno. Esta diferencia de temperatura permanece casi constante hasta $580^{\circ} \mathrm{C}$, la cual corresponde a una temperatura de horno de $\sim 500^{\circ} \mathrm{C}$ dependiendo de la presión parcial de propano. Al aumentar el flujo de propano se ha observado que aumenta la temperatura real de la pila debido a que se activa la reacción de oxidación parcial o reformado del hidrocarburo (propano). El voltaje a circuito abierto (O.C.V.) y la densidad de corriente bajan a cero unos pocos grados por encima de 
dicha temperatura de pila, pero las propiedades eléctricas pueden recuperarse de nuevo bajando la temperatura del horno, si el incremento no es demasiado elevado. Se cree que la descomposición del LSCO ocurre a mayor temperatura en estas condiciones reductoras. Probablemente este comportamiento es debido a que el cátodo produce también $\mathrm{CO}_{2}$ e $\mathrm{H}_{2}$ a partir de la reacción de oxidación del propano, lo que provoca la disminución de las diferencias de presiones parciales de $\mathrm{H}_{2}$ y $\mathrm{O}_{2}$ entre ambos electrodos, las cuales son las responsables de generar la fuerza electromotriz de la pila. La resistencia total de la pila cae hasta unos pocos kohms durante el calentamiento a unos $300-320^{\circ} \mathrm{C}$ dependiendo del espesor del electrolito y de la superficie pintada del cátodo. Se ha encontrado de 1 a $3 \mathrm{k} \Omega$ para un electrolito de $20 \mu \mathrm{m}$ y una superficie del cátodo de $10 \mathrm{~mm}^{2}$.

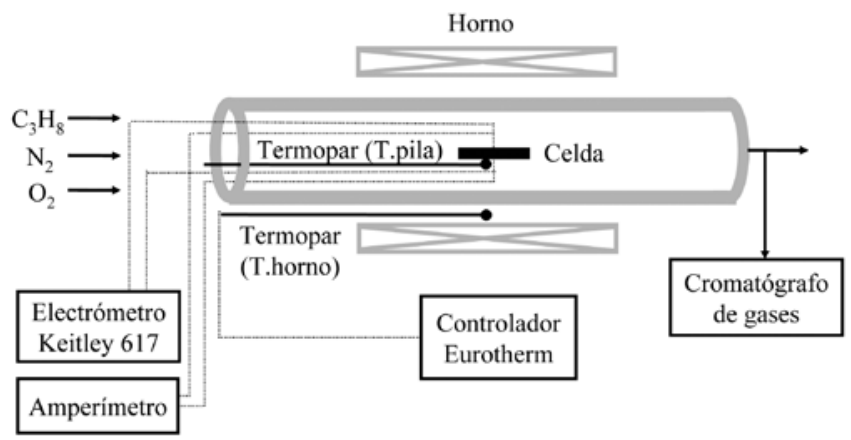

Fig. 5- Esquema del reactor utilizado para la evaluación de las propiedades electroquímicas de las pilas de combustible preparadas.

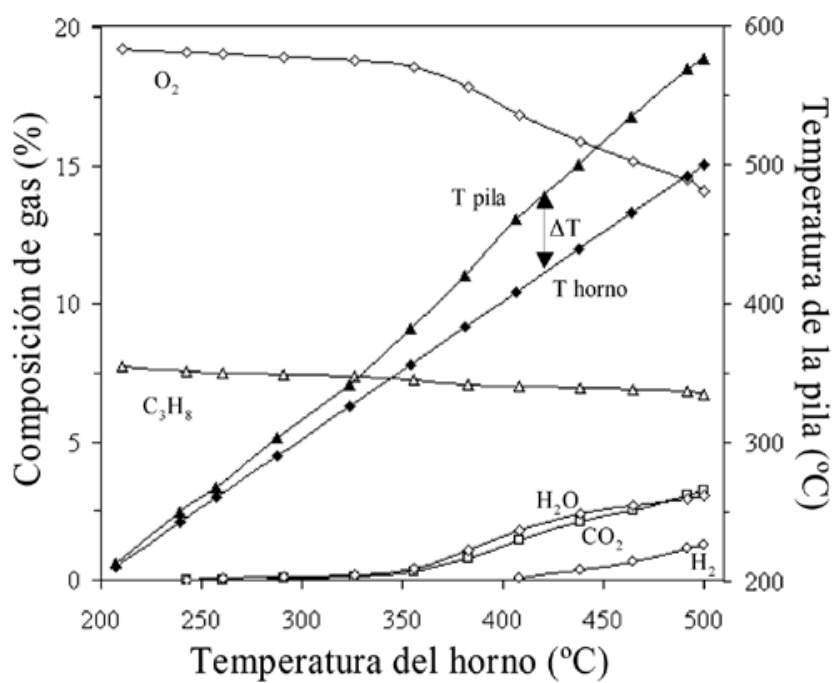

Fig. 6- Análisis cromatográfico de los gases resultantes de una pila de combustible soportada sobre el ánodo operando en circuito abierto.

En otra serie de experimentos, se ha estudiado la influencia de la velocidad del flujo del gas portador (aire sintético) en la temperatura y en las propiedades eléctricas de la pila para una temperatura constante del horno. En la figura 7 se muestra la densidad de corriente y la dependencia con la temperatura de una pila de combustible con un cátodo activo de $10 \mathrm{~mm}^{2}$ calentado en el horno a una temperatura constante de $500^{\circ} \mathrm{C}$. La temperatura del horno y la densidad de corriente siguen un comportamiento similar, es decir, ambas aumentan con el incremento de velocidad de flujo total. La reacción se activa con la velocidad de flujo total probablemente porque el mecanismo de la reacción está controlado por la difusión de los gases. Sin embargo el O.C.V. no es sensible a la variación de velocidad de flujo total (ver figura 8).

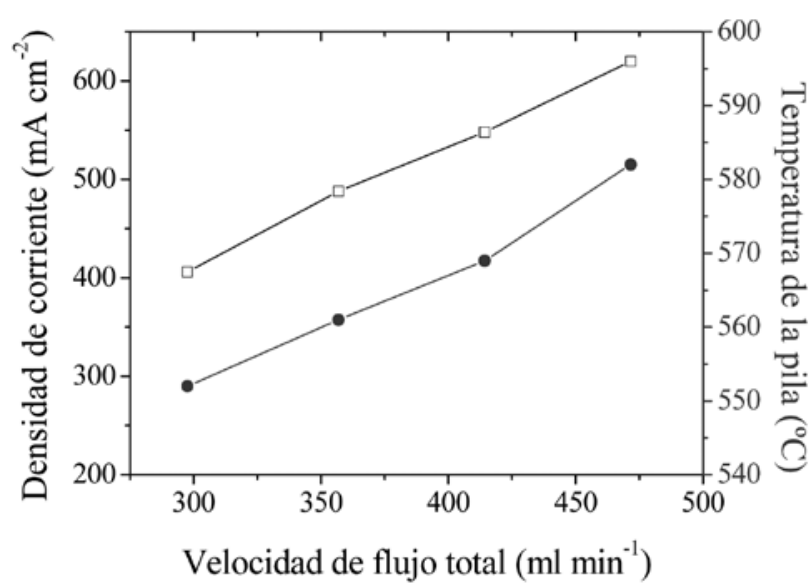

Fig. 7- Temperatura y densidad de corriente en función de la velocidad del flujo total para una pila de combustible soportada en el ánodo de una sola cámara.

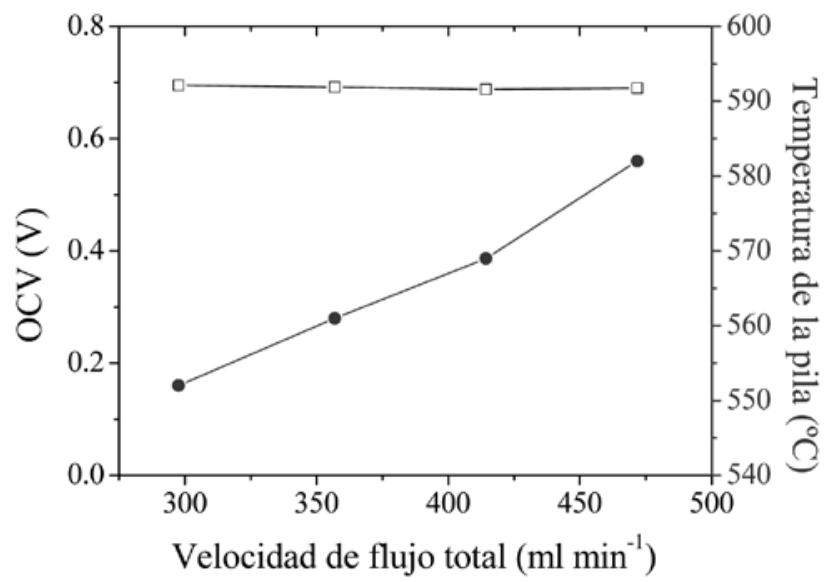

Fig. 8- Temperatura y voltaje en circuito abierto (O.C.V.) en función de la velocidad del flujo total para una pila de combustible soportada en el ánodo de una sola cámara.

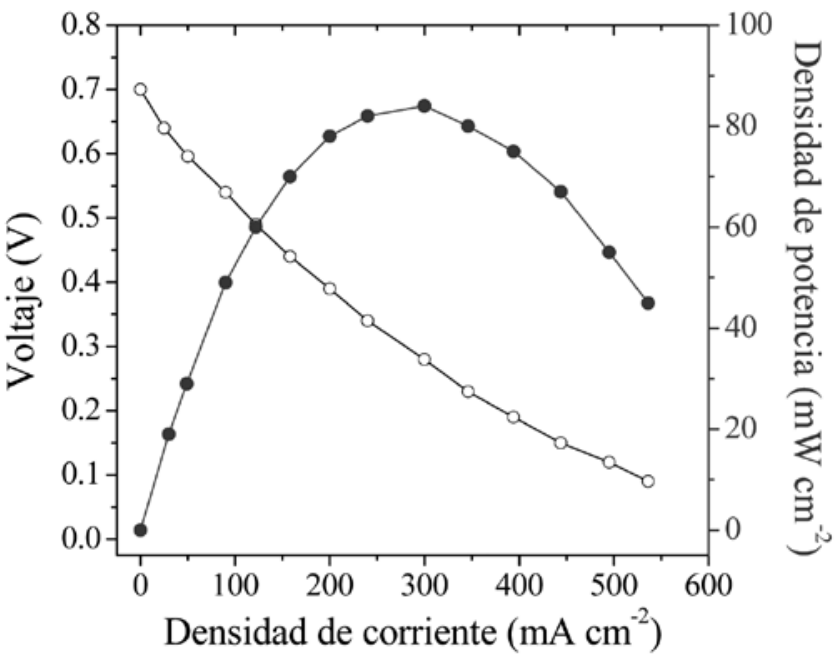

Fig. 9- Voltaje y densidad de potencia en función de la densidad de corriente para una pila soportada en el ánodo de una sola cámara medida a $590^{\circ} \mathrm{C}$. 
El pico de la densidad de potencia para una pila operando a $590^{\circ} \mathrm{C}$ que corresponde a una temperatura de horno de $500^{\circ} \mathrm{C}$ se representa en la figura 9. Las pilas de combustible muestran las mismas características eléctricas después de varias horas de reacción, incluso calentando y enfriando la pila de combustible cíclicamente. En esta figura se puede observar que la densidad de potencia alcanza un máximo de $84 \mathrm{~mW}$ $\mathrm{cm}^{-2}$ a una densidad de corriente de $300 \mathrm{~mA} \mathrm{~cm}^{-2}$. El voltaje a circuito abierto es de $\sim 700 \mathrm{mV}$, valor que es sensiblemente inferior al encontrado para las pilas soportadas sobre el electrolito. Probablemente este comportamiento ocurre por la existencia de algunos defectos que se han formado cuando las partículas del electrolito de CGO no se han distribuido correctamente sobre la superficie del ánodo o en la misma tinta. Entonces, aparecen algunas grietas y aglomerados en la superficie de la capa del electrolito y las propiedades eléctricas de las pilas de combustible decrecen drásticamente (ver figuras 10 y 11). Las grietas se forman durante el tratamiento térmico de densificación a elevadas temperaturas y dependen de los parámetros reológicos de la tinta tales como su viscosidad o el tamaño de grano de las partículas. El ánodo de Ni/CGO y el cátodo de LSCO/CGO parecen estables en estas condiciones experimentales. Sin embargo, el comportamiento a mayor
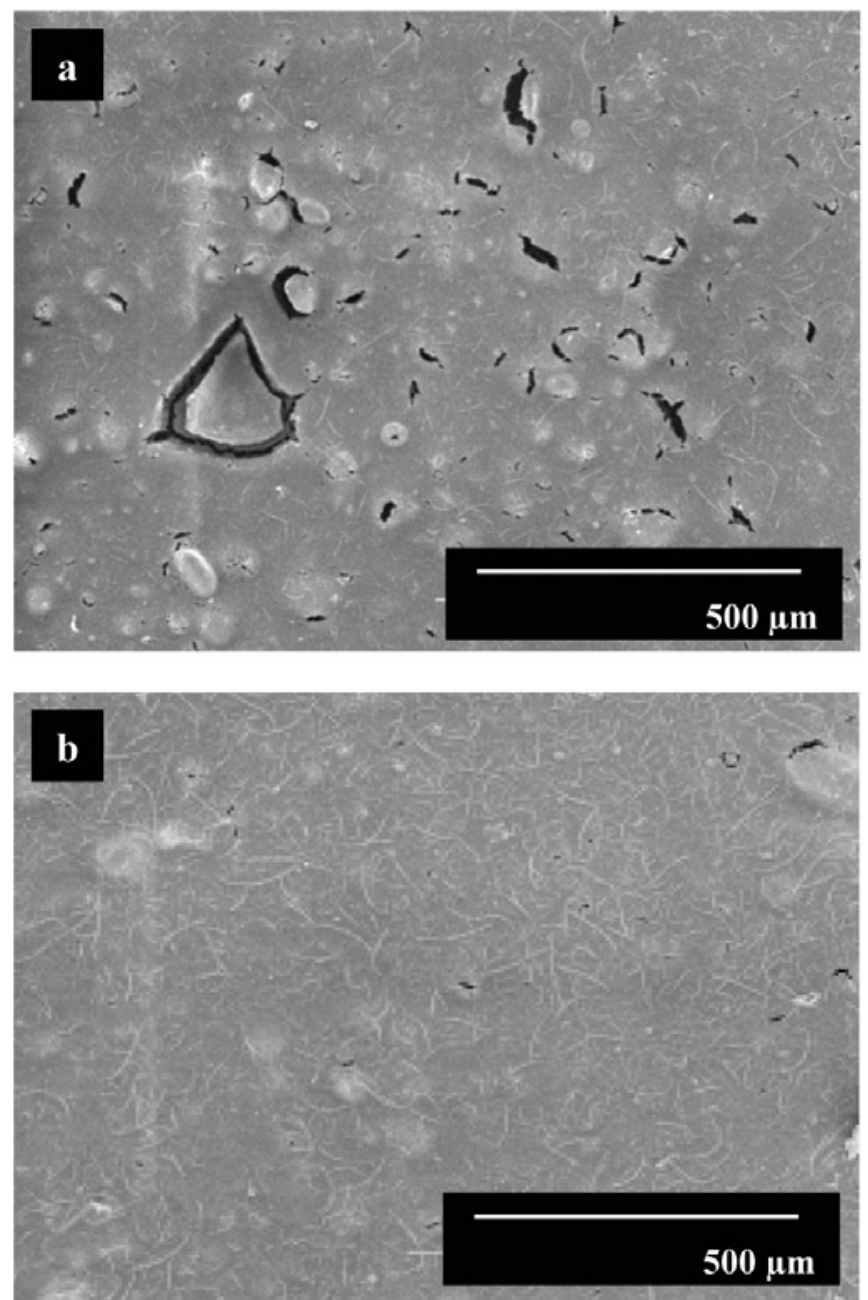

Fig. 10- Micrografías SEM de las superficies de dos electrolitos con diferentes densidades de defectos. Las grietas se han formado durante el proceso de densificación a alta temperatura.

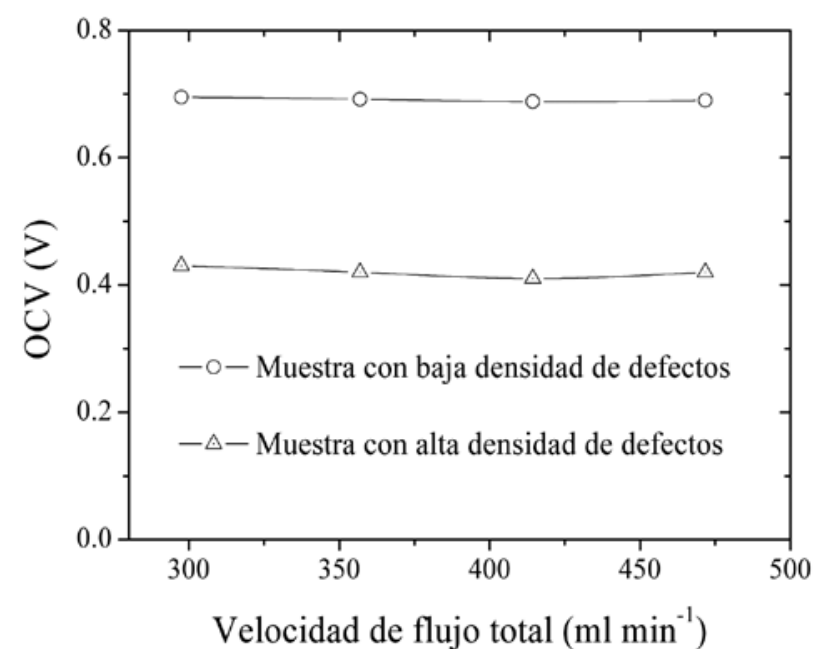

Fig. 11- Voltaje en circuito abierto (O.C.V.) de dos pilas de combustible soportadas en el ánodo con diferentes densidades de defectos en los electrolitos de CGO.

tiempo y las propiedades cíclicas deben ser estudiadas con mayor detalle para determinar la verdadera estabilidad de la pila.

Por otra parte, se ha investigado la dependencia del O.C.V. y la densidad de corriente próxima a $\mathrm{V} \sim 0$ con la velocidad de flujo de propano. Se ha encontrado que sólo hay un estrecho rango de la velocidad de flujo de propano donde las propiedades eléctricas son óptimas. Unos pocos $\mathrm{ml} / \mathrm{min}$ por encima o por debajo de la concentración óptima de propano disminuyen drásticamente el O.C.V., la densidad de corriente de la pila y la máxima de densidad de potencia (ver figura 12). Este comportamiento es muy diferente al de las pilas de combustible de electrolito soportado de una sola cámara, dónde es necesaria una presión parcial crítica de propano para iniciar la reacción, pero una vez la reacción se ha iniciado, la variación de la densidad de potencia con respecto al propano

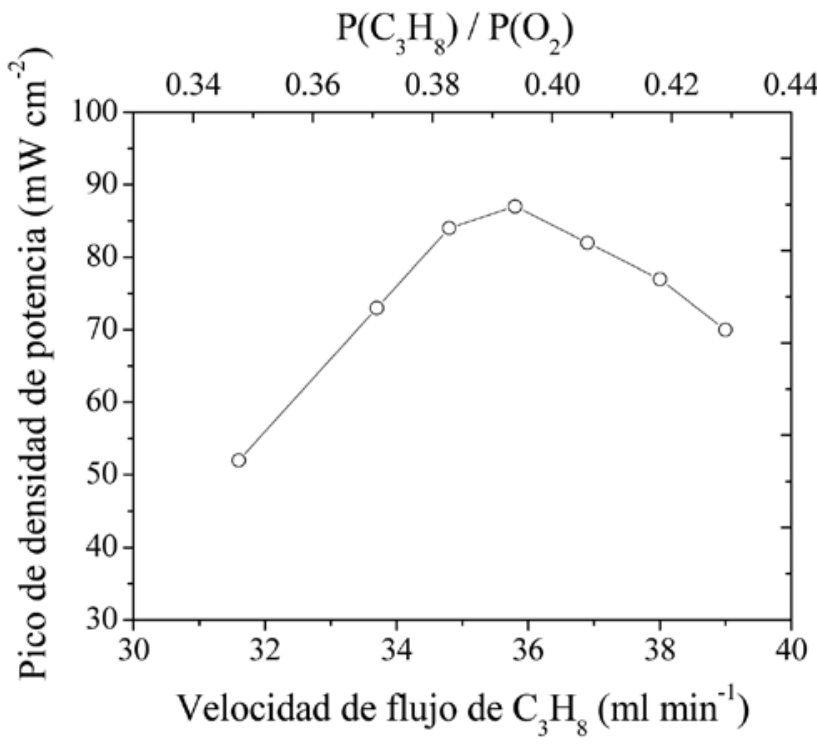

Fig. 12- Pico de densidad de potencia en función de la velocidad de flujo de propano y de la relación de presiones parciales propano-oxígeno, para una velocidad de flujo de aire constante $(442 \mathrm{ml} / \mathrm{min})$ y una temperatura de horno constante de $500^{\circ} \mathrm{C}$. 
parece ser cero o ligeramente negativa (17). Probablemente, esta diferencia se deba al espesor y porosidad del ánodo. Cuando la porosidad del ánodo no es suficientemente buena entonces hay una fuerte dependencia con la presión parcial de propano. Actualmente, se está trabajando activamente en el análisis cromatográfico de los productos de la reacción para poder comprender los mecanismos involucrados en este tipo de pilas de combustible.

\section{CONCLUSIONES}

Las pilas de combustible de una sola cámara soportadas en el ánodo son una prometedora alternativa para obtener elevadas densidades de potencia a relativamente bajas temperaturas porque permiten reducir considerablemente el espesor del electrolito. Sin embargo, es difícil obtener una buena calidad de capa de electrolito y una óptima porosidad para el ánodo al mismo tiempo. El $\mathrm{La}_{0,5} \mathrm{Sr}_{0,5} \mathrm{CoO}_{3-\delta}$ (LSCO) es un cátodo estable en atmósfera de propano diluido en aire para pilas de combustible de óxido sólido de una sola cámara basadas en electrolito soportado sobre el ánodo, hasta temperaturas de trabajo próximas a $650^{\circ} \mathrm{C}$. En las pilas de combustible soportadas en el ánodo de una sola cámara es posible aumentar la temperatura de la pila aumentando el aislamiento del horno. Por tanto, el calentamiento producido por la oxidación del propano, la cual es mucho más exotérmica que la oxidación del hidrógeno (reacciones 1 y 2) puede ser usado para mantener las condiciones operacionales de las pilas de combustible. Las propiedades eléctricas dependen notablemente de la velocidad de flujo de gas transportador y de la presión parcial de propano. Este comportamiento probablemente se debe a que el mecanismo de la reacción está controlado por la difusión de los gases.

\section{AGRADECIMIENTOS}

Este trabajo se ha realizado con los recursos del proyecto del plan nacional MCyT MAT2003-04556 y MAT2006-11080C02-02.

\section{BIBLIOGRAFÍA}

1. W. van Gool, Possible use of surface migration in fuel cells and heterogeneous catalysis, Philips Res. Repts., 20, 81-93 (1965).

2. G.A. Louis, J.M. Lee, D.L. Maricle, J.C. Trocciola, Solid electrolyte electrochemical cell, US Patent, 4248941 (1981).

3. C.K. Dyer, A novel thin-film electrochemical device for energy conversion, Nature 343, 6258, 547-548 (1990).

4. P. Moseley, D. Williams, Mixed reactant fuel cells with flow through porous electrodes, Nature 346, 23-31 (1990).

5. T. Hibino, H. Iwahara, Simplification of solid oxide fuel cell system using partial oxidation of methane, Chem. Lett., 7, 1131-1134 (1993).

6. I. Riess, P.J.van der Put, J. Schoonmam, Solid oxide fuel cells operating on uniform mixtures of fuel and air, Solid State Ionics, 82, 1-4 (1995).

7. T. Hibino, S. Wang, S. Kakimoto, M. Sano, Single chamber solid oxide fuel cell constructed from an yttria-stabilized zirconia electrolyte, Electrochemical and Solid-State Letters, 2, 7, 317-319 (1999).

8. T. Hibino, T. Hashimoto, T. Inoue, J.I. Tokuno, S.I. Yoshida, M. Sano, A lowoperating-temperature solid oxide fuel cell in hydrocarbon-air mixtures, Science, 288, 5473, 2031-2033 (2000).

9. Z. Shao, S.M. Haile, J. Ahn, PD. Ronney, Z. Zhanand S.A. Barnett, A thermally self-sustained micro solid-oxide fuel-cell stack with high power density, Nature, 435, 7043, 795-798 (2005).

10. Z. Shao, S.M. Haile, A high-performance cathode for the next generation of solid-oxide fuel cells, Letters to Nature, 431,7005, 170-173 (2004).

11. B.C.H. Steele, Appraisal of $\mathrm{Ce}_{1-y} \mathrm{Gd}_{\mathrm{y}} \mathrm{O}_{2-\mathrm{y} / 2}$ electrolytes for IT-SOFC operation at $500^{\circ} \mathrm{C}$, Solid State Ionics $129,1-4,95-110(2000)$.

12. L. Minervini, M.O. Zacate, R.W. Grimes, Defect cluster formation in $\mathrm{M}_{2} \mathrm{O}_{3}$ doped $\mathrm{CeO}_{2}$, Solid State Ionics 116, 3-4, 339-349 (1999).

13. H. Yahiro, K. Eguchi, H. Arai, Electrical properties and reducibilities of ceria-rare earth oxide systems and their application to solid oxide fuel cell, Solid State Ionics 36, 71 (1989).

14. K. Eguchi, T. Setoguchi, T. Inoue, H. Arai, Electrical properties of ceriabased oxides and their application to solid oxide fuel cells, Solid State Ionics 52, 1-3, 165-172 (1992).

15. I. Riess, D. Braunshtein, D.S. Tannhauser, Density and ionic conductivity of sintered cerium oxide-gadolinium oxide $\left[\left(\mathrm{CeO}_{2}\right)_{0.82}\left(\mathrm{GdO}_{1.5}\right)_{0.18}\right]$, J. Am. Ceram. Soc., 64, 8, 479-485 (1981).

16. D. Pérez-Coll, D. Marrero-López, P. Núñez, S. Piñol, J.R. Frade, Grain boundary conductivity of $\mathrm{Ce}_{0.8} \mathrm{Ln}_{0.2} \mathrm{O}_{2-\delta}$ ceramics $(\mathrm{Ln}=\mathrm{Y}, \mathrm{La}, \mathrm{Gd}, \mathrm{Sm})$ with and without Co-doping, Electrochim. Acta, 51, 28, 6463-6469 (2006).

17. S. Piñol, Stable single-chamber solid oxide fuel cells based on doped ceria electrolytes and $\mathrm{La}_{0.5} \mathrm{Sr}_{0.5} \mathrm{CoO}_{3}$ as a new cathode, Fuel Cells Science and Technology, 3, 4, 434-437 (2006). 